\title{
Retention of environmental habituation in rats as a function of the environment during the retention interval
}

\author{
TIMOTHY R. THOMAS \\ Alma College, Alma, Michigan 48801
}

\begin{abstract}
The time course of female rats' habituation to familiar test environments was measured with a resident-intruder paradigm. A resident was permitted $0,2,5,10$, or $30 \mathrm{~min}$ of environmental adaptation prior to introduction of an intruder, and a dominance test was then conducted in which both rats were allowed to compete for drops of water. The degree of dominance which the resident attained was a function of the duration of adaptation, reaching a maximum after $5 \mathrm{~min}$. The retention of this habituation was investigated as a function of the type of retention environment and the duration of the retention interval. Retention intervals of 5 or $25 \mathrm{~min}$ in unfamiliar environments produced significant loss of habituation, while the same intervals in familiar environments did not produce habituation loss. The degree of similarity between habituated and retention environments did not appear to be an important variable affecting retention. When the retention interval was extended to $25 \mathrm{~h}$ in a familiar retention environment, no environmental habituation was retained.
\end{abstract}

After a rat enters an environment, a short period of time usually elapses before consummatory behaviors such as eating, drinking (Bolles \& Rapp, 1965), or sexual behavior (Thomas, 1973) are displayed at full strength. These observations imply that a period of environmental habituation, during which the rat investigates the environment, must occur before other motives may become prepotent. In addition, Chance and Mead (1955) have shown that when a rat is removed from a familiar environment and returned after some time has elapsed, some habituation is lost and must be reacquired. The major purpose of the present study is the identification of factors responsible for this loss of environmental habituation.

In order to measure the extent of environmental habituation, an operational definition of this concept is required. It must be based on an observable behavior that changes reliably and meaningfully with time, following entrance to an environment. The superior operation will be one that yields minimum random variation between and within Ss. Social dominance may provide a reliable definition of environmental habituation since the extent of dominance would be expected to change with the acquisition and loss of environmental habituation. In the present study, a thirsty resident, given prior adaptation to an environment, is allowed to compete for drops of water with an equally thirsty intruder. If drinking dominance, defined by the number of drops of water drunk by the resident as compared to the number drunk by the intruder, is directly related to duration of the resident's prior adaptation, then drinking dominance will serve as a measure of environmental habituation. A preliminary purpose of the present study is to determine the validity and reliability of this method of measuring the course of environmental habituation.

\section{METHOD}

\section{Subjects}

The Ss were 30 adult female Sprague-Dawley rats, housed in pairs, with food continuously available. All Ss had been used weekly for 10 weeks, in an introductory psychology laboratory, to demonstrate basic operant-conditioning phenomena such as discrimination training, secondary reinforcement, and extinction. After the bars had been removed, the test chambers used in that laboratory were used in the present experiment. While the majority of each S's learning experience was in a single test chamber, frequent substitution of components and occasional switching of chambers gave most rats exposure to more than one test chamber. Therefore, prior to the start of this experiment, each rat was allowed to press a bar in each of the six chambers, and each rat was given dipper training trials in each chamber after new bedding and a mixture of rat feces from the home cages had been placed under the floor of the chambers.

\section{Apparatus}

Three types of experimental environment were used: (1) The test chambers were six $8 \times 11 \times 8 \frac{1}{4}$ in. clear-plastic-sided metal boxes with metal rod floors. A tray containing sawdust and rat feces was placed under the floor of each cage. On one wall, a 2-in-diam hole permitted access to a metal tray on which a drop of water could be placed by the operation of a solenoid. The sound of the solenoid served as a discriminative cue for the presence of water. (2) The home cage of each $S$ was a $7 \times 7 \times 9 \frac{1}{2}$ in. metal-sided cage with a wire front and floor. (3) The novel boxes were two 12-in semicircular observation boxes with sheet metal walls, glass fronts, wooden tops, and wooden floors covered with sawdust bedding.

\section{Procedure}

Twelve groups, each made up of seven different pairs of rats were used. To form the different pairs, the 30 Ss were divided into two squads of 15 rats each. On each testing day, seven pairs were made up from within one squad. The remaining unpaired rat was not tested on that day. Pairing was done randomly with the restrictions that the same pair never be formed twice, that cagemates never be paired, and that the same rat never be used twice in the same group. There was no systematic order in which the 12 groups were tested. None of the seven pairs tested on a single day were assigned to the same group. All members of one 


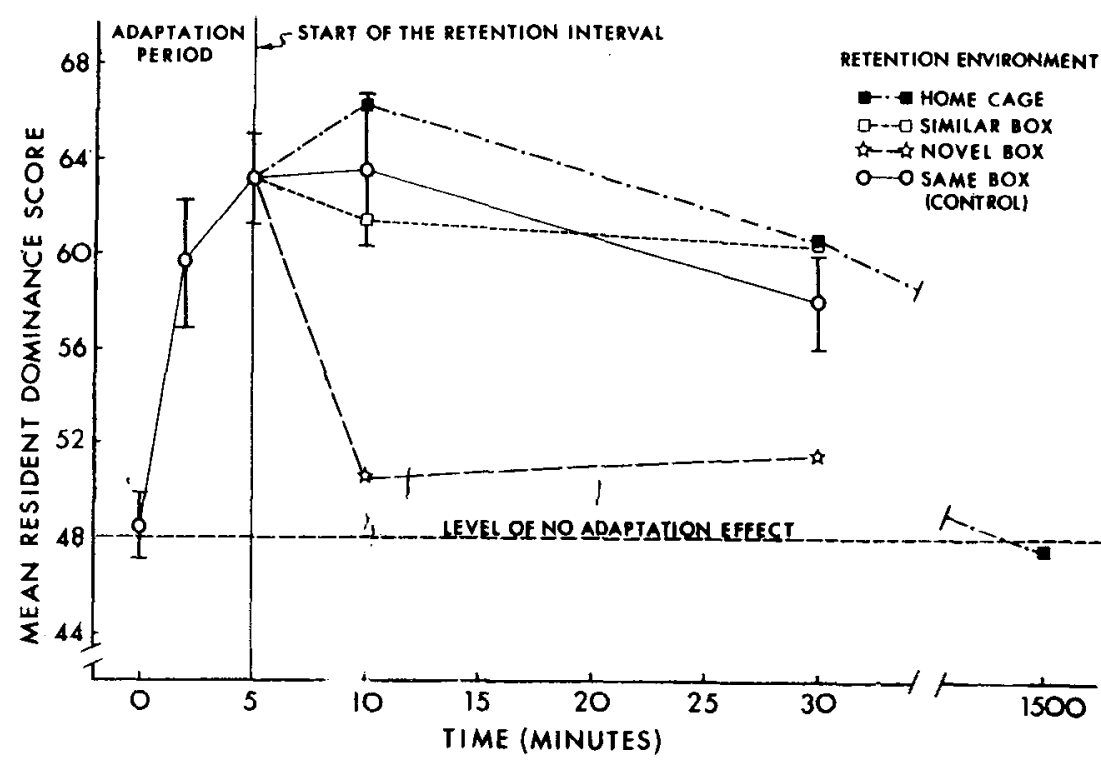

Fig. 1. Mean resident dominance score for the 12 different groups. Brackets around the five control groups, which were only briefly removed from the test environment, indicate \pm 1 standard error of the mean. The 5 - and 25-min retention intervals fall on the abscissa at 10 and $30 \mathrm{~min}$, since the retention interval was added to the 5 -min adaptation period.

squad were deprived of water 23 to $25 \mathrm{~h}$ before testing, and were given at least $24 \mathrm{~h}$ access to water following testing. Thus, neither squad was tested on consecutive days. At the start of each testing day, Ss to be tested were given 10 drops of water in a test chamber which was not to be used on that day. All testing was done at night, when the level of extraneous noise was at a minimum.

Habituation Testing. Five groups were used to determine the effect of length of adaptation on the outcome of competition for water. Two animals which met the above restrictions were paired, designated A or B by marking the rump with ink, and assigned to a $0-, 2-, 5-, 10-$, or $30-\mathrm{min}$ adaptation group. The $\mathrm{A}$ member was then placed alone in one test chamber for the assigned adaptation period and was termed the resident. The B member, termed the intruder, was brought to the test chamber from her home cage, a distance of about $15 \mathrm{ft}$. The resident was then removed and both rats were held for $7 \mathrm{sec}$ before being placed together in the test chamber. Five seconds later, the drinking dominance test began. It consisted of presenting, at 5 -sec intervals, 24 individual drops of water, and recording which tat drank each drop. Ss were awarded 2 points for each drop of water drunk. When neither rat drank before the next drop was given, or when both rats replaced each other over the tray so quickly that the $\mathrm{E}$ could not determine which had drunk the water, each rat was awarded 1 point. At the end of this drinking dominance test, both animals were returned to their home cages for a 25- to 30-min intertest interval. Subsequently, the same procedure was repeated with a different test chamber and with the roles of the A and B Ss reversed. Thus, the B member became the adapted resident and the $A$ member the intruder. Following the second drinking dominance test, the number of points received by the resident in both tests was totalled and was termed the resident dominance score. The range of possible scores was 0 to 96 , scores below 48 indicating intruder dominance and those above 48 indicating resident dominance.

In the case of the 0-min adaptation group, for which the resident was given no prior adaptation, the designation as resident or intruder was arbitrary. Individual differences between pair members do not affect the resident dominance score since each rat serves equally as resident and as intruder. Drinking motivation should not have changed appreciably during the two tests since these Ss did not begin to show any decrement in operant-response rate with the size drop used until approximately 100 drops had been consumed.

Retention Testing. Six groups were used to determine the effect of different environments on retention of environmental habituation. A procedure similar to that used with the 5 -min adaptation group, described above, was employed. After a pair was selected, the $A$ member was given 5 min of adaptation to one of the test chambers, and was termed the resident. Then, rather than beginning the drinking dominance test to determine the extent of habituation attained in $5 \mathrm{~min}$, the test was delayed until the resident had spent a retention interval alone in another environment. The level of habituation attained at the start of the retention interval was independently determined by the 5-min adaptation group. The six groups were formed by assigning pairs to retention intervals of 5 or $25 \mathrm{~min}$ in one of three different retention environments: (1) the S's home cage, (2) one of the novel boxes, or (3) another of the similar test chambers. During both the adaptation period and the retention interval, the intruder remained in her home cage with her cagemate. At the end of the retention interval, the resident and the intruder were transported to the test chamber in which the resident had previously adapted and held for $7 \mathrm{sec}$. A drinking dominance test was then begun. At the end of the test, both rats were returned to their home cages for the intertest interval. The same pair was then retested, using the same procedure, but with different experimental environments of the same type and with the resident-intruder roles reversed.

A last group was used to determine whether there was any effect of the 5-min adaptation to the test chamber remaining after $25 \mathrm{~h}$ in the home cage. For this group, several modifications of the above retention testing procedure were required: (1) the resident was with her cagemate during the retention interval, (2) both resident and intruder were permitted only $1 \mathrm{~h}$ of access to water in their home cages immediately after the resident's adaptation period, and (3) the group was tested during 3 consecutive days at the end of the experiment.

\section{RESULTS}

The solid line in Fig. 1 plots the mean resident dominance score as a function of the length of adaptation to the test chamber prior to the drinking dominance test. When no prior adaptation was permitted, the score did not differ significantly from 48 , the level expected when neither resident nor intruder is dominant. After $2 \mathrm{~min}$ of adaptation, the resident achieved significant drinking dominance $(p<.01)$ and maintained this dominance after each of the longer adaptation times.

For the purpose of analyzing the retention data after $5 \mathrm{~min}$ of adaptation, the 10 - and 30 -min adaptation groups were conceptualized as control groups, revealing 
the level of resident dominance retained after 5 or 25 additional minutes in the same environment. The retention data were then analyzed with a 2 by 4 factorial analysis of variance, where the factors were duration of the retention interval (either 5 or $25 \mathrm{~min}$ ) and type of retention environment (the same, a similar, a novel, or the home cage environment). Neither the main effect of duration nor the interaction was significant. The main effect of type of retention environment was significant $(\mathrm{F}=12.22, \quad \mathrm{df}=3 / 48, \quad \mathrm{p}<.001) . \quad$ A subsequent Dunnett's test revealed that only the novel environment produced a loss of adaptation significantly different from that of the control groups $(p<.01)$. Figure 1 shows the means of each group in this analysis.

The mean resident dominance score of the group which was tested for retention after $25 \mathrm{~h}$ in the home cage is shown in Fig. 1. This retention interval produced no significant retention of the $5 \mathrm{~min}$ of adaptation to the test chamber.

Typical behavior observed during the dominance test consisted of one rat's placing herself in front of the water tray and drinking each drop as it was presented. The other rat would explore other regions of the chamber and, on occasion, attempt to displace her partner from the advantageous position. These attempts usually consisted of a side-stepping approach and a struggle, involving primarily the forepaws and the nose, wherein one rat would force the other to remove her head from the hole to the water tray. These struggles were normally of short duration, ending when one rat would leave the tray area to investigate other areas of the chamber. On occasion, a rat would leave the tray area without any interaction with the other rat, and both would explore, engage in social behavior, or self-groom.

The effects of adaptation time could be observed because, on the average, it was the intruder who was both more likely to lose the aggressive encounter and to spontaneously leave the tray region. In a typical case from the 5-min adaptation group, Pair Member A drank 15 of the 24 drops when she was the resident, and 8 when she was the intruder. Pair Member B drank 16 drops as resident and 8 as intruder. One drop was assigned to neither member since it was presented during a vigorous struggle and neither rat was able to drink before the next drop was given. Thus, a resident dominance score of 63 was recorded for this pair. No rat ever succeeded in drinking all 24 drops presented. The maximum number of drops drunk by one rat during a session was 22 . When this rat was the intruder, she succeeded in drinking only 17 drops, thus producing a resident dominance score of 58 for the pair. It may be assumed that this rat had higher social dominance rank than her partner since her partner was rarely able to displace her from the region of the tray. This pair had been assigned to the 30-min adaptation group, and the effects of the adaptation can be seen in the reduced number of drops drunk by the socially dominant subject after the submissive $\mathrm{S}$ had been given prior adaptation.

\section{DISCUSSION}

The resident-intruder paradigm, as used in this experiment, was shown to be a valid method of measuring the course of environmental habituation. Thus the amount of prior adaptation is an important determinant of the outcome of dominance tests when competition for water is observed. Since the test environments were very familiar to all $\mathrm{Ss}$, the curve obtained should be considered one of rehabituation. If novel test environments had been used, the habituation curve might be expected to take longer to. reach asymptote than the $5 \mathrm{~min}$ required in this experiment.

Because the analysis was based on behavioral differences between roles, either resident or intruder, not on the behavior of individual rats, the method used provided a powerful control for between-S differences. Thus any difference between pair members' social dominance rank did not mask the effect of adaptation. Furthermore, the method permitted determination of curves using relatively few Ss since many different pairs were formed using the same Ss.

Differences between the resident and intruder in the probability of exploratory behavior could account for the resident's dominance in the drinking dominance tests. After the adaptation period during which the resident investigates the test chamber, the resident would be less likely to leave the area of the tray and explore the chamber, and so would be more likely to drink than would the exploring intruder. Furthermore, when the rats were actually struggliing to displace each other from the water tray, the intruder's tendency to explore would account for her tendency to break off the encounter more readily than the resident. This analysis is derived directly from the common observation that a rat's exploratory behavior decreases as time in a test environment increases (Berlyne, 1960).

The environment during the retention interval was shown to be an important determinant of the amount of habituation retained. Environments familiar to the rat, e.g., the home cage or a similar test chamber, did not produce any significant loss of adaptation within $25 \mathrm{~min}$, while the novel retention environment produced almost total loss of the effect of adaptation within $5 \mathrm{~min}$. This result cannot be attributed to the dissimilarity between the test and novel environments since the home cage was also dissimilar to the test environment. Yet, with the same retention interval, it produced no habituation loss. A retention environment which is both similar to the test environment and yet unfamiliar to the rat, may not be available since the stimulus generalization which makes the environments similar would also be expected to produce a degree of familiarity. Thus, the results of Chance and Mead (1955), which indicated that the degree of retention of habituation was a function of the amount of stimulus generalization, are not in disagreement with these results; rather, the present data indicate that the distinction between familiarity and similarity is a valid one, and that the familiarity 
dimension is the more important determinant of habituation loss.

It is possible to interpret the effects of the novel environment as a process of dishabituation (Lynn, 1966). This interpretation would view the effects of adaptation as the habituation of a generalized orienting reflex. The novel environment would present new, dishabituating stimuli which would serve to restore the reflex and thus destroy the effects of adaptation. This view emphasizes the importance of the novel stimuli themselves and could account for the loss of adaptation occurring after $25 \mathrm{~h}$, but not $25 \mathrm{~min}$, in the home cage by the increased likelihood that dishabituating stimuli, such as noises arising from normal laboratory activity, would occur during the longer interval. An alternative view of the effects of the novel environment might emphasize the arousing characteristics of novel stimuli. The dissipation of the increased arousal induced by the novel environment would reinforce exploratory behavior in the test chamber (Berlyne, 1960) and thus produce the loss of dominance. This view must regard the loss of adaptation after $25 \mathrm{~h}$ in the home cage as a passive decay of some memory trace.

\section{REFERENCES}

Berlyne, D. E. Conflict, arousal and curiosity. New York: McGraw-Hill, 1960.

Bolles, R. C., \& Rapp, H. M. Readiness to eat and drink: Effect of stimulus conditions. Journal of Comparative \& Physiological Psychology, 1965, 60, 93-97.

Chance, M. R. A., \& Mead, A. P. Competition between feeding and investigation in the rat. Behavior, 1955, $8,174-182$.

Lynn, R. Attention, arousal and the orientation reaction. New York: Pergamon Press, 1966.

Thomas, T. R. Use of a resident-intruder paradigm to measure the influence of environmental adaptation on mounting behavior. Psychological Record, 1973, 23, 405-416.

(Received for publication June 11, 1973; revision received April $9,1974$. 